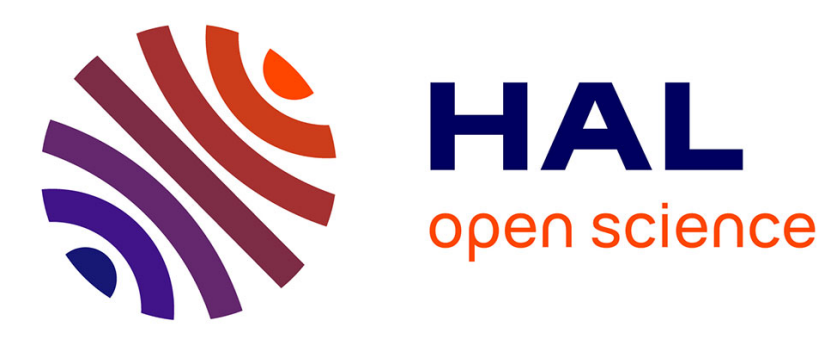

\title{
Water: A medium where dissipative structures are produced by a coherent dynamics
}

Nadia Marchettini, Emilio del Giudice, Vladimir Voeikov, Enzo Tiezzi

\section{To cite this version:}

Nadia Marchettini, Emilio del Giudice, Vladimir Voeikov, Enzo Tiezzi. Water: A medium where dissipative structures are produced by a coherent dynamics. Journal of Theoretical Biology, 2010, 265

(4), pp.511. 10.1016/j.jtbi.2010.05.021 . hal-00608941

\section{HAL Id: hal-00608941 \\ https://hal.science/hal-00608941}

Submitted on 16 Jul 2011

HAL is a multi-disciplinary open access archive for the deposit and dissemination of scientific research documents, whether they are published or not. The documents may come from teaching and research institutions in France or abroad, or from public or private research centers.
L'archive ouverte pluridisciplinaire HAL, est destinée au dépôt et à la diffusion de documents scientifiques de niveau recherche, publiés ou non, émanant des établissements d'enseignement et de recherche français ou étrangers, des laboratoires publics ou privés. 


\section{Author's Accepted Manuscript}

Water: A medium where dissipative structures are produced by a coherent dynamics

Nadia Marchettini, Emilio Del Giudice, Vladimir Voeikov, Enzo Tiezzi

PII: S0022-5193(10)00256-0

DOI: doi:10.1016/j.jtbi.2010.05.021

Reference: YJTBI 6000

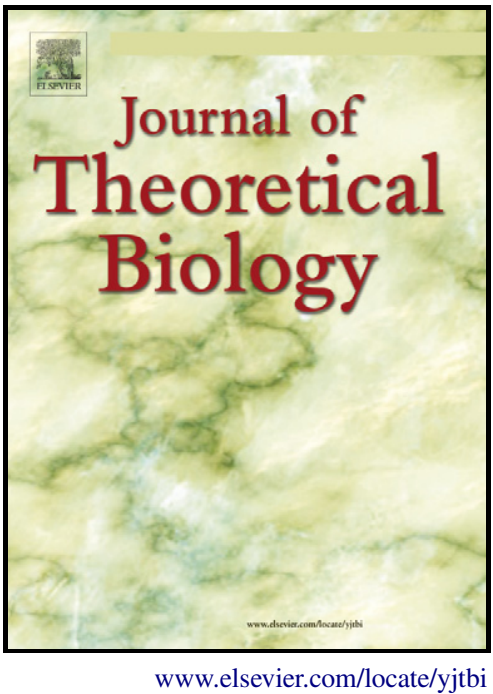

To appear in: $\quad$ Journal of Theoretical Biology

Received date: $\quad 20$ November 2009

Revised date: $\quad 17$ May 2010

Accepted date: $\quad 17$ May 2010

Cite this article as: Nadia Marchettini, Emilio Del Giudice, Vladimir Voeikov and Enzo Tiezzi, Water: A medium where dissipative structures are produced by a coherent dynamics, Journal of Theoretical Biology, doi:10.1016/j.jtbi.2010.05.021

This is a PDF file of an unedited manuscript that has been accepted for publication. As a service to our customers we are providing this early version of the manuscript. The manuscript will undergo copyediting, typesetting, and review of the resulting galley proof before it is published in its final citable form. Please note that during the production process errors may be discovered which could affect the content, and all legal disclaimers that apply to the journal pertain. 


\title{
Water: a medium where dissipative structures are produced by a coherent dynamics
}

\author{
Nadia Marchettini ${ }^{a}$, Emilio Del Giudice ${ }^{\mathrm{b}}$, Vladimir Voeikov ${ }^{\mathrm{c}}$ and Enzo Tiezzi ${ }^{\text {*a }}$ \\ ${ }^{a}$ Department of Chemistry, University of Siena, via della Diana 2a, 53100, Siena, Italy. Fax +39 0577 232012; Tel: \\ +390577232012; E-mail:marchettini@unisi.it; tiezzienzo@unisi.it \\ ${ }^{b} I N F N$, Via Celoria, 16, 20133, Milan, Italy and International Institute of Biophysics, Landestiftung Hombroich, \\ Raketenstation, Kapellener Str. o.M, D-41472, Neuss, Germany. Fax +3902 50317208; Tel: +3902 50317734; E- \\ mail: emilio.delgiudice@mi.infn.it \\ ${ }^{c}$ Department of Bio-organic Chemistry, Faculty of Biology, Lomonosov Moscow State University, Moscow, 119992, \\ Russia and International Institute of Biophysics, Neuss, Germany. Fax/Tel: +7 (495) 939 1268; E-mail: \\ v109028v1@yandex.ru
}

\begin{abstract}
The Belousov-Zhabotinsky phenomenon is analyzed in a framework where the dynamics of dissipative structures outlined by Prigogine is implemented through the collective dynamics produced in liquid water by Quantum Electrodynamics, which has received recently some experimental support. A mechanism allowing the appearance of self-produced oscillations is suggested.
\end{abstract}

Keywords: water; dissipative structures; coherent domains.

\section{Introduction}

The problem how to account for the emergence of the highly ordered structures observable in Nature (in particular in the biosphere) out of the unordered crowd of independent atoms/molecules has been addressed in modern science along two pathways: the thermodynamics of irreversible processes (TIP) pioneered by Prigogine and the quantum field theory (QFT).

The first approach has produced the discovery of the dissipative structures that are highly complex structures which are open systems able to self-organize, thus producing order out of chaos, and give rise to dramatic 
decreases of entropy. This discovery occurred in a conceptual framework that looks at the system as a whole and extracts from its behaviour in space and time a global principle that characterizes its dynamics.

The second approach has been able to show that quantum interaction among elementary objects, through the breakdown of the symmetries of the Lagrangian, is able to produce extended regions where the phases of the elementary components get correlated. This loss of independence curtails sharply the number of microstates producing the decrease of entropy.

The two above discoveries open the way to a fascinating perspective; could the extended coherent regions discovered by QFT be the dynamical basis of the dissipative structures discovered by TIP?

We wish to address this problem in the present paper in the particular case of an ordered system, the Belousov-Zhabotinsky (BZ) reaction [1]. The BZ reaction can occur within many different set-ups, as illustrated in a recent paper [2] giving rise to the appearance of ordered space patterns exhibiting well-defined time evolution. The emergence of such structures is connected with the dynamically induced break-down of the symmetries existing in the original ensemble of elementary components. This break-down of symmetry is the consequence of the collective dynamics generated by the boundary conditions whose emergence is described in thermodynamics by the concept of negentropy introduced by Schrödinger [3] and developed by Szent-Györgyi [4], Prigogine [5] and the Siena group [6].

The BZ reaction exhibits some peculiar features that, inside a more complex frame, are present also in the living process. An important recent result [7] is the discovery that the occurrence of the BZ reaction in a particular layout where lamellar lipid structures are involved demands the presence of liquid water whose weight should exceed a critical threshold ( $70 \%$ of the weight of the lipid) whereas neither water nor lipids are chemical reactants in the reaction. Their role should then be connected to the creation of the conditions of the selforganizing capability of the system [8]. Below a threshold of hydration this capability could not arise; it arises above a critical threshold. The aim of this paper is to try to shed some light on this surprising result. However, this result could appear less surprising considering that the presence of water has been found long ago to be necessary in a number of chemical processes including the combustion [9].

The analysis presented here mainly deals with the experimental layout used in Ref. [7]. Different layouts investigated in the literature could involve different dynamics. Consequently the proposal presented in this paper does not replace, but adds up to the dynamical ingredients governing the very complex phenomenon, known as BZ-reaction. 


\section{Dissipative structures (DS) and QED coherence in liquid water}

In this section we summarize some information on water needed for our analysis. Water is the most abundant substance on Earth and has been extensively studied; a number of model structures have been proposed and refined in the conventional framework of molecular physics governed by quantum mechanics. However, it remains an anomalous liquid in as far as no single model has been able to explain all of its properties. All these models share the property that water molecules are assumed to be interacting through static short-range forces. However this tenet should not be universally true since extended regions of organized water had been detected in aqueous systems mainly at interfaces.

As early as in 1957 Albert Szent-Györgyi [4] wrote that by 1947 up to that date up to 175 papers were published devoted to water hydrating hydrophilic surfaces. They showed that the zone of hydrating water (interfacial water) may have the thickness of tens and hundreds of molecule layers and not just a few as usually believed by many physicists and chemists. This result emerged from the consideration of the luminescent properties of different dyes dissolved in water and depended strongly on the physical state of water, as structured by the different states of polarization of the surface. Based on the full set of these experimental results, SzentGyörgyi was able to suggest that the thick layers of "organized" water adjacent to the hydrophilic surfaces could allow very long lasting electronic excitations that could play a significant role in the energy transfer near the cell surfaces, where water resides in a state similar to a liquid crystal, as stressed also by Chandrasekhar [10]. The above considerations suggest strongly that a fruitful approach to self-organization is a joint task of chemists, biologists and physicists that help each other to get rid of the respective prejudices [8].

Same decades later, just after 2000, the suggestion of Szent-Györgyi has been corroborated by G. Pollack and his Seattle group [11].

They were able to find, by using microspheres and dyes as probes, that very thick layers of water appear on hydrophilic surfaces, whose depth can reach many microns (sometimes up to 500!). In these regions dyes and other solutes cannot penetrate, so that they are called exclusion zones (EZ).

EZ water is more viscous than normal water and is able to release electrons in a significant amount, so that EZ water has a non negligible (up to hundreds of millivolts) redox potential with respect to bulk water or adjacent aqueous solution; EZ water is thus a reducing species whereas normal water is an oxidant. The layers of EZ water should play an important role in the chemical interactions occurring on their surface. Moreover differences of electric potential (about tens of millivolts) appear on the interface EZ water- normal water. 
These results find a theoretical corroboration in a recently proposed theory of liquids based on the concepts of Quantum Electrodynamics (QED). Referring the reader to the Refs. [12-16] we summarize here the main results of this approach:

1) At room temperature, liquid water consists of a mixture of coherent and incoherent water. At $0^{\circ} \mathrm{C}$ the percentages are $50 \%$ and $50 \%$; at $30{ }^{\circ} \mathrm{C}$ they are $40 \%$ coherent, $60 \%$ incoherent [14].

2) Coherent water is organized in coherence domains, whose size is about $1000 \AA(0.1 \mu \mathrm{m})$, each containing about 5.5 millions molecules that oscillate, in phase with an electromagnetic field trapped in the domain, between two configurations of the molecular electron cloud: the fundamental one in which electrons are strongly bound (ionization potential $12.60 \mathrm{eV}$ ) and an excited configuration $(12.06 \mathrm{eV})$ in which one electron per molecule is "quasi-free" and is therefore susceptible of further excitation.

The coherent state is the superposition of two molecule states, the molecule ground state having the statistical weight 0.87 and the excited state having the statistical weight 0.13 . This means that at any time there are 0.13 quasi-free electrons per molecule, i.e. 700,000 quasi-free electrons, in the coherence domain.

The external surface of the domain is permanently polarized with the negative charges on the outside, so that for an isolated domain, an electrical "membrane" potential of about 100 millivolts is generated! If domains coalesce, as in normal water, these potentials neutralize each other.

When there are isolated domains, electrons have a chance to come out through the quantum tunnel effect or, possibly, a mild external excitation. This property could account for the huge release of electric charge from the water droplets suspended in the clouds, which is at the origin of lightning.

3) In bulk water the electromagnetic oscillations and the thermal fluctuations couple mutually, so that each molecule can shift in time from the coherent to the non-coherent regime and viceversa. The array of CDs of bulk water is then flickering and CDs cannot exhibit in a permanent way their features.

However, near a hydrophilic surface, the attraction CD-surface protects the CDs from the thermal assaults stabilizing them. Water gets totally coherent (glass state) at about $200 \mathrm{~K}$, so that an attraction corresponding to a decrease of the effective temperature by one hundred of Kelvins would be enough [16]. By the way, the Pollack's estimate [11] of the effective temperature existing in the EZ zone is much lower than that of bulk water.

4) If coherent and incoherent water are separated in space, a redox pile up to hundreds of millivolts is created, in which coherent water, the electron donor, is the negative pole (reducing element) and incoherent water the positive pole (oxidizing element) [17].

5) By virtue of its quasi-free electrons, a coherence domain of water has unique properties with respect to all other liquids. It has a rich spectrum of excited states corresponding to vortices of quasi-free electrons. These 
vortices have angular momentum (quantized at integer values) and similarly quantized magnetic moments (this property explains why turbulent water can be discovered by animals and humans, whose nervous systems are known to be very sensitive detectors of magnetic fields). In the presence of external fields, such as the magnetic field of the Earth, these vortices line up and are summed.

Since electrons move coherently, they do not collide, which means that the vortices are cold, and in the absence of internal friction caused by collisions, their life is very long (weeks, months, years). Because of their persistence, it is possible to sum a large number of excitations, producing energies as large as one wishes starting from individual low-energy excitations, provided that they are below the ionization threshold. The coherence domain of water is therefore a structure capable of transforming low-grade (high entropy) incoherent environmental energy into high-grade (low entropy) coherent energy, that can excite specific chemical reactions.

When the energy stored in the electromagnetic field of a coherence domain becomes equal to (resonates with) the energy of activation of a specific non aqueous molecule captured in the domain by the fact that it resonates at the oscillation frequency of the domain (the criterion for biomolecules), the domain discharges, transferring its energy to the single molecule that receives a specific excitation (enzyme activation) that makes it capable of performing a specific chemical reaction (coupling of electromagnetic and chemical modes).

If the domain is immersed into an energy field, it recharges again and a permanent oscillation is created in which charging is electromagnetic and discharge is chemical. As a result the dissipative structure introduced by Prigogine is supported by the QFT also [18].

The above QED predictions have found important experimental corroboration. In reference [19] the properties of liquid water at low temperature, when the liquid is in a glassy state, are successfully accounted for quantitatively. Moreover evidence has been found [20] of the coexistence in liquid water of two phases having different densities. Finally evidence of the existence in the aqueous surroundings of biomolecules of e.m. fields playing a significant dynamical role has been reported by the Nobel Prize Luc Montagnier [21]. He was able to detect the emission of electromagnetic signals in the aqueous structures surrounding highly diluted bacterial DNA, showing therefore that water plays an active dynamical role.

\section{Analysis of the BZ reactions}

Let us address now the problem of the BZ reaction, describing first the TIP framework and discussing at the end the possible dynamical role of water.

The basic mechanism of morphogenesis, namely differentiation of tissues from stem cells, was proposed 50 years ago by Turing [22], who pointed out that if morphogens obey a reaction-diffusion equation, they may 
undergo symmetry-breaking transitions. This, in turn, generates spatially organized states, now known as Turing structures, which may explain the initial stages of cell aggregation and development and epigenesis. In particular, if we consider a generic reaction-diffusion system:

$$
\frac{\delta C_{i}}{\delta t}=D_{i} \nabla^{2} C_{i}+f_{i}\left(\ldots, C_{i}, C_{j}, \ldots\right)
$$

where $C_{i}$ is the concentration of the $\mathrm{i}^{\text {th }}$ species participating in the reaction, $f_{i}$ is the nonlinear kinetics equation describing the reaction and $D_{i}$ is the diffusion coefficient of species i. Assuming that eq.(1) has a homogeneous steady state solution, $f_{i}\left(C_{s}\right)=0$, let us consider the evolution of a small perturbation $c_{s}$ around $C_{s}$ and separate it in Fourier space,

$$
c_{s}=\sum_{k} a_{s k} e^{\lambda_{k} t+i \bar{k} \cdot \bar{r}}
$$

where $\lambda_{k}$ is the growth rate of the mode with a wave vector $k$. Substituting eq.(2) in eq.(1) and retaining only the linear term,we obtain an eigenvalue equation for $\lambda_{k}$ for the linear operator

$$
L_{i j}=F_{i j}-D_{i} k^{2} \delta_{i j}
$$

where $F_{i j}$ is the Jacobian matrix of the kinetic function $f_{i}$.

For a certain nonzero mode k, Turing instability occurs when the real part of the eigenvalue $\lambda_{k}$ of operator (3) becomes positive, so that the homogeneous steady state becomes unstable and the system undergoes a transition from the homogeneous state to a patterned state. Turing structures are characterized by a constant wavelength and 
time-independent behaviour. They are generated when the diffusion coefficients of the morphogens (in the simplest case one activator and one inhibitor) are very different.

Target patterns where pulses are emitted periodically from the same leading centre (pacemaker) have been observed in a host of reaction-diffusion systems that generate chemical oscillations. These systems occasionally produce more complex structures, e.g. outward rotating spirals [23].

The simultaneous presence of propagating waves and stationary patterns was found when the BZ reaction was carried out in the anisotropic environment of a lipid/water binary system. In order to mimic the biological complexity of a cell environment, the Siena Ecodynamics group studied the BZ oscillator in the aqueous compartment of different phospholipid/water lamellar phases [7, 24-27].

All the lipids studied are major components of plasma membranes. Lipids in water are a good model for biological environments, since different aqueous domains in living systems are usually separated by a membrane where the basic structural unit is a single phospholipid bilayer where both surfaces facing water are hydrophylic.

In this system we get the appearance of a time dependent symmetry breaking pattern of reactant concentrations through the amplifications, produced by different rates of diffusion, of the gradients present at a given time. However, we have discussed in the previous section that on hydrophilic surfaces there are gradients of energy able to drive the motion of different species in water. We ask ourselves if, in the layout of Ref. [7], where lamellar structures water-lipids are present, these gradients of energy could induce a self-organizing dynamics able to contribute, possibly in cooperation with other dynamics, to the emergence of a pattern of reactant concentrations.

Figure 1 shows a typical Turing structure obtained in the Siena laboratory.

\section{< FIGURE 1 >}

The intriguing result obtained in the Siena laboratory was the disappearance of all the BZ patterns and structures when the (water phase)/(lipid) ratio was low. For water concentrations below $70 \%$ weight/weight with respect to lipid, no self-organization phenomena were observed. Since all known forms of life and all physiological solutions have a water content in the range $60-99 \%$ (i.e. water is the molecule of life), our findings could open new perspectives in the understanding of life mechanisms, especially with respect to the role of water in self-organizing systems (dissipative structures) $[2,5,7,24]$. 
The big question is whether there is a unified theory for the ways in which elements of a system organize themselves to produce behaviours typical of large classes of systems. In complex systems, we accept that processes occurring simultaneously at different scales or levels are important and coupled together, and the intricate behaviour of the whole system depends on its units in a nontrivial way. Description of the whole system's behaviour requires a qualitatively new theory [8] because the laws governing this behaviour are qualitatively different from those governing the individual units.

Dissipative Structures maintain their non-equilibrium thermodynamic state thanks to a continuous dissipation of energy toward the environment. The order produced by this dissipation generates new order and new organization (autocatalytic structures) but if the dissipation is interrupted or diminished, the structure can collapse and may not return to its initial state (irreversibility) $[5,8]$.

The system thus self-organizes in virtue of internal non-linear processes that guarantee a balance between the entering energy and the exiting energy [8], maintained by the variation of entropy occurring within the dissipative structure. The low grade energy picked up from the environment is transformed with a corresponding decrease of entropy within the dissipative structure into high-density energy able to perform "useful" work, such as collective molecular excitations. This energy produces work on the environment, inducing in the same time an increase of entropy in it that compensates the decrease of entropy which occurred within the dissipative structure.

Let us now discuss how the presence of water and in particular of coherent-EZ water described in the previous Section could contribute to the self-organization of the system.

The BZ-system has initially a number of interfaces (the Petri dish, the interfaces among different component phases present in the emulsion and so on), many of them hydrophilic, so that a pattern of EZ thick layers is present.

Correspondingly, on the surface of the EZ-layers gradients of electric potential should appear according to the QED theory and to the experimental findings of the Pollack group [11]. The formation of the EZ-layers, that have a well defined depth dependent on the polarization of the hydrophilic surface, demand a well defined amount of water, that should correspond just to the observed critical threshold in the hydration. The theoretical framework we are suggesting should allow to estimate the value of the critical threshold. We hope to do that in future research.

Let us outline now the dynamics occurring on the surface of EZ-coherent water. We recall from Ref. [14] and [16] the main physical parameters of coherent water.

$$
\text { Coherent fraction } F_{c}(\mathrm{~T})=0.4 \quad \text { at } \mathrm{T}=300 \mathrm{~K}
$$


Density of coherent water $=0.92$

Molecule density $=3 \times 10^{22} \mathrm{~mol} / \mathrm{cm}^{3}$

Number of quasi-free electrons per molecule $=0.13$

Volume of the coherence domain $\mathrm{CD}$ at $\mathrm{T}=0$

$V=8\left(\frac{1}{2} 10^{-5}\right)^{3} \mathrm{~cm}^{3}=10^{-15} \mathrm{~cm}^{3}$

Volume of the (spherical) $\mathrm{CD}$ at $\mathrm{T} \neq 0=4 / 3 \pi\left(\mathrm{R}_{0}-\delta\right)^{3}$

Within the coherent region an e.m. field is trapped, whose energy density

$$
U=\text { const } . A^{2}
$$

is proportional to the square of the vector potential $\vec{A}$ of the trapped e.m. field (see Ref. [14]).

Out of the coherent region the e.m. field falls off exponentially so that on the boundary there is a strong gradient $\nabla A^{2}$ and consequently, for eq. (5), $\nabla U \neq 0$.

This gradient gives rise to a force, acting on the present particles having charge $Q$ and mass $M$ (ponderomotive force) $[28]$

$$
\vec{F}=\frac{-Q^{2}}{M} \nabla A^{2} \propto \frac{-Q^{2}}{M} \nabla U
$$

that is repulsive and inversely proportional to $M$. Thus in a molecule electrons are repelled tens of thousands times more strongly than nuclei. Molecules thus get stretched and acquire an electric dipole moment, whose negative charge is outwards.

As a consequence a charge double layer appears on the surface of coherent water where the external layer is negatively charged.

According to the definition of the coherent fraction 


$$
F_{c}(T)=\frac{0.92 \frac{4}{3} \pi\left(R_{0}-\delta\right)^{3}}{8 R_{0}^{3}}=0.92 \frac{\pi}{6}\left(1-\frac{\delta}{R_{0}}\right)^{3}
$$

we get, recalling eqs. (4), $\delta=20 \AA$ that the depth $2 \delta$ of the boundary region is $40 \AA$.

The capacity of the interface layer per unit surface $\frac{C}{S}$ is

$$
\frac{C}{S}=\frac{\varepsilon_{r} \varepsilon_{0}}{2 \delta} \cong 20 \mu \mathrm{Farad} / \mathrm{cm}^{2}
$$

In order to estimate the amount of electric charge per unit surface, we recall (Ref. [29]) that the quasi-free electron settles in the molecule $0.35 \AA$ out of the electron core in the ground state, so that we can assume that during the dynamical process of excitation-deexcitation this distance could be included in the range $0.35 \div 0.70 \AA$. Recalling eqs. (4) we get

$$
\frac{Q}{S} \cong 1.2 \div 1.8 \mu \mathrm{C} / \mathrm{cm}^{2}
$$

so that the potential difference of the double layer is enclosed in the range:

$$
55 m V \leq \Delta V=\frac{\frac{Q}{S}}{\frac{C}{S}} \leq 110 m V
$$

whose agreement with the observed membrane potentials is intriguing. 
In this way the energetic excitations of the coherent interfacial water give rise to a variable gradient of the electric potential, that in turn governs the rate of inflow of the reactants.

We can write the fundamental relation between the surface electric potential and the density of energy released to the water

$$
\nabla V=\text { const } . \nabla U
$$

In this way the excitation of coherent water induced by the output of chemical reactions increases the electric potential barrier, reducing the inflow of reactants and a fluctuating rate of chemical reactions appears.

Moreover the molecules present in the region settle according to the profiles of the electric potential and the possibility of patterns arises, supporting the Turing dynamics.

The inflow of the BZ reactants occurs now not in a homogeneous space, but in a volume where the existence of the EZ-water on the surfaces produces a spatial pattern of the non-coherent fraction of water, where solutes are dissolved. This pattern depends on electric potential and redox potential gradients that are affected in turn by the energy produced in the chemical reactions. This energy is mainly absorbed by the coherent water (the energy transfer toward coherent regions is much faster than the transfer toward non-coherent objects), whose inner structure changes, by modifying in turn the potential gradients.

We get thus a non linear self-consistent dynamics.

Reactants flow in the system not in a diffusive random way but along the paths corresponding to the steepest gradients, then react chemically; the energy produced, absorbed by the EZ-water, modifies the coherent structure changing the pattern of the potential gradients, that in turn change the rate of flow of the reactants, that consequently change the rate of reaction. A scheme for ordered fluctuations thus arises, both in space and time. Let us explore an extreme case. Suppose that the rate of the BZ-reaction, enhanced by the electron excitations available on the EZ-water, becomes so high that the EZ-water is put out of coherence and loses its potential structure. Then the rate of reaction would decrease dramatically because the inflow of reactants is no longer driven by the potential gradients but left to diffusion.

Consequently the inflow of energy that pushed water out of coherence is stopped and QED dynamics can restore in a very short time the original coherence and the rate of reaction starts again to increase. We have got an oscillation! 
The suggested interplay between the quantum electrodynamics of interfacial water and the chemical dynamics of the BZ reactants opens a promising outlook. We conclude by observing that the patterns of electric and redox potentials on the water interfaces that constrain the independence of single molecules and funnel them along preferred pathways are nothing else that the electrodynamical implementation of the thermodynamic concept of negentropy whose formulation is the combination of the insights of the physicist Schrödinger, the biologist SzentGyörgyi and the chemist Prigogine.

\section{Water and spatially ordered structures}

In this section we discuss how a liquid emerging from the QFT dynamics outlined above could produce spatially ordered (Turing) structures. A CD of water is an extended region where all molecules are in a minimum entropy state as a consequence of a common oscillation on a frequency whose value is defined by the electrodynamics of molecules, chemically non interacting. The coherent feature of this state guarantees that its entropy is fairly low.

Molecules of species other than water can be admitted within the CD provided that they are able to oscillate on the same frequency, without changing the self-governing electrodynamical structures of the CD. This is possible only if these host molecules are a small number, say less than $1 \%$ of the water molecules, so that their QED regime within the CD can be determined by perturbation theory. Different than the water molecules, these other molecules can bind each other by several and different chemical or electrostatic forces, so that their oscillation frequency depends on the strength of such bindings, that usually depends in turn on the geometry. The prescription that the host molecules should resonate with the water molecules in order to minimize entropy implies that the geometry should be well-defined. A dynamical condition, the electrodynamical coherence, produces then geometrical order!

Moreover, the existence of a rich spectrum of excited levels of CDs, each one with its specific oscillation frequency, implies that the geometrical order could evolve in time and produce also regular oscillations according to the oscillations of the CDs between their configurations. The role of the water CDs is thus fundamental and the necessity of its presence for the occurrence of the Turing structures can be understood, along the above lines.

Thus water gives rise to the emergence of order out of chaos, as in the case of the above described BZ experiments. 
By virtue of the above oscillations, a set of coherence domains may in turn become coherent (coherence of coherence domains), giving rise to a living (pulsing) organism consisting maily of water molecules organized in coherence domains, resonating with a small number of biomolecules residing in less organized water.

\section{Conclusion}

The discussion of the present paper applies to the layout of Ref. [7], whereas different layouts could involve different dynamics, as described in the existing literature. The experimental finding in Ref. [7] of a critical relationship between the amounts of water and lipids forming lamellar structures indicates that both the formation of EZ-water due to the presence of hydrophilic surfaces and existence of less organized aqueous phase in the same reaction system is the condition for the appearance of order out of chaos in chemical processes proceeding in aquatic systems.

\section{References}

1 B.P. Belousov, A Periodic Reaction and Its Mechanism in Sbornik Referatov po Radiatsionni Medtitsine, $1958,145$.

2 F. Rossi, S. Ristori, M. Rustici, N. Marchettini and E. Tiezzi, J. Theor. Biol., 2008, 255, 404-412.

3 E. Schrodinger What is Life? Cambridge Univ. Press, 1944.

4 A. Szent-Györgyi, Bioenergetics, Academic Press, New-York, 1957.

5 I. Prigogine, Thermodynamics of Irreversible Process, Wiley, New York, 1955.

6 A. Facchini, H. Kantz and E. Tiezzi, Physical Review E, 72, 021915, 2005; selected for publication in the Virtual Journal of Biological Physics Research.

7 A. Magnani, N. Marchettini, S. Ristori, C. Rossi, F. Rossi, M. Rustici, O. Spalla and E. Tiezzi, J. Am. Chem. Soc., 2004, 126 (37), 11406-11407.

8 E. Tiezzi, The Essence of Time, WIT press, Southampton, 2003, pp. 123 and the foreword by Ilya Prigogine therein.

9 W.A. Bon, J. Chem. Soc. London., 1931, 338-361.

10 S. Chandrasekhar, Liquid Crystals, Cambridge University Press, 1992.

11 Jian-ming Zheng, Wei-Chun Chin, E. Khijniak, E. Khijniak Jr., G. H. Pollack, Adv. Colloid. Interface Sci., 2006, 127, 19-27 and papers quoted therein.

12 G. Preparata, QED Coherence in Matter, World Scientific Publishing, 1995.

13 E. Del Giudice, G. Preparata and G. Vitiello, Phys. Rev. Lett., 1988, 61, 1085-1088. 
14 R. Arani, I. Bono, E. Del Giudice, and G. Preparata, Int. J. Mod. Phys. B, 1995, 9, 1813-1841.

15 E. Del Giudice and A. Tedeschi, Electromagn. Biol. Med., 2009, 26, 48-54.

16 M. Buzzacchi, E. Del Giudice and G. Preparata, Int. J. Mod. Phys. B, 2002, 16 (25), 3771-3786.

17 V. L. Voeikov, V. V. Koldunov and D. S. Kononov, Kinetics and Catalysis, 2001, 42, 606-608.

18 E. Del Giudice, R.M. Pulselli and E. Tiezzi. Ecological Modelling, 2009, 220, 1874-1879.

19 C.A. Yinnon and T. Yinnon, Modern Physics Letters B, 2009, 23, 1959-1973.

20 C. Huang et al., Proceedings of the National Academy of Sciences USA 2009, 106, 15214-15218.

21 L. Montagnier et al., Interdisciplinary Science Computer Life Sci 2009, DOI: 10.1007/s12539-009-0036-7.

22 A. M. Turing, Philos. Trans. Roy. Soc. London B, 1952, 327, 37-52.

23 R. Kapral and K. Showalter, eds., Chemical Waves and Patterns, Kluwer, Dordrecht, NL, 1995.

24 N. Marchettini, S. Ristori, F. Rossi and M. Rustici, International Journal of Ecodynamics, 2006, 1, 55-63.

25 G. Biosa, M. Masia, N. Marchettini and M. Rustici, Chem. Phys., 2005, 308 (1-2), 7-12.

26 S. Ristori, F. Rossi, G. Biosa, N. Marchettini, M. Rustici and E. Tiezzi, Chem. Phys. Lett., 2007, 436, 175-178.

27 M. A. Budroni, M. Masia, M. Rustici, N. Marchettini, V. Volpert and P. C. Cresto, J. Chem. Phys., 2008, 128 (11) 1102.

28 A. Einstein and J. Laub, Annalen der Physik (Leipzig), 1908, 26, 541.

29. E. Del Giudice, A. Galimberti, L. Gamberale and G. Preparata, Mod. Phys. Lett. B, 1995, 9, 953. 
Figure captions.

Figure 1. Lipid/BZ system: Turing structures 


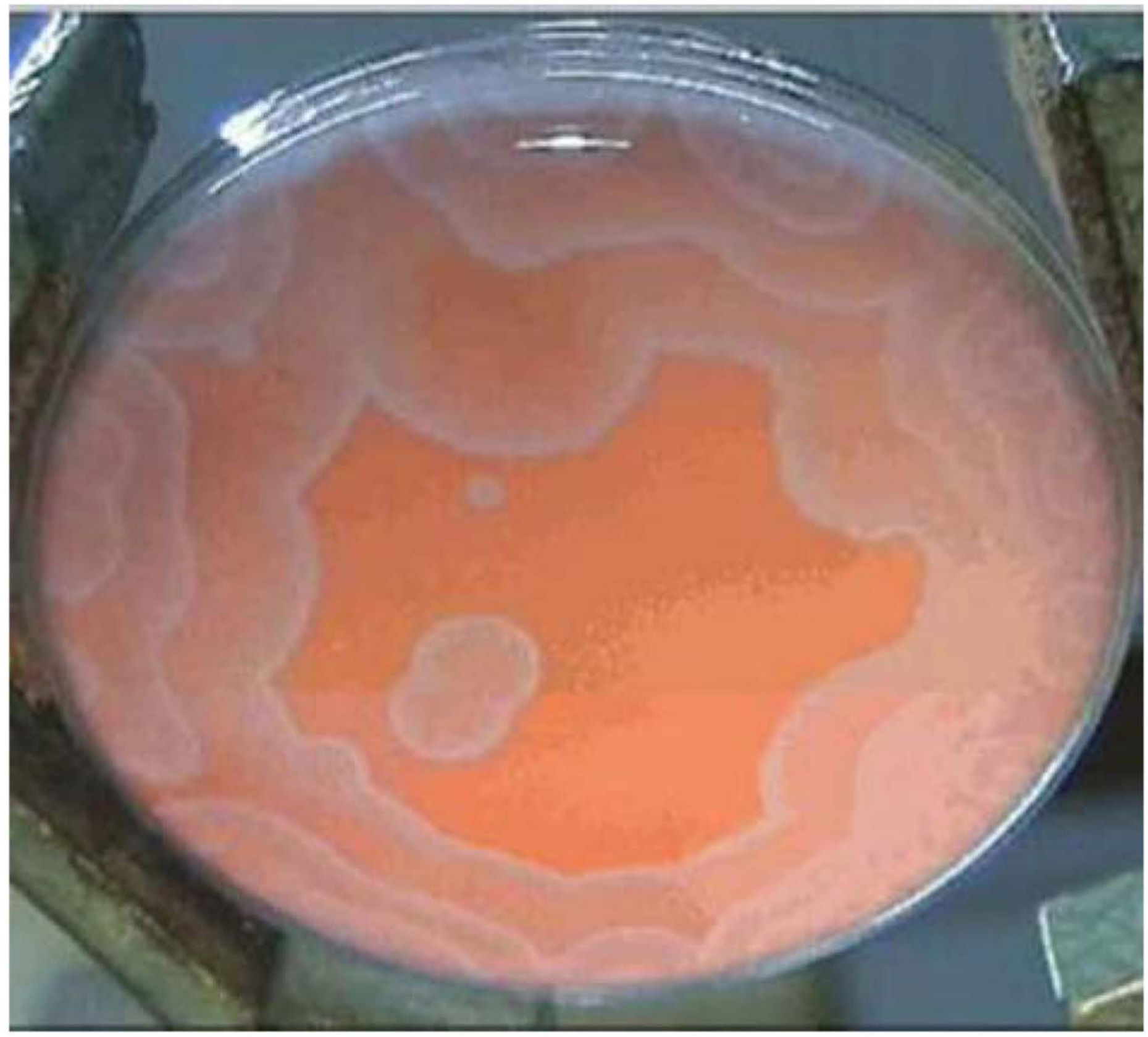

